\title{
Personalized Persuasive Technology - Development and Validation of Scales for Measuring Persuadability
}

\author{
Marc Busch $^{1}$, Johann Schrammel $^{1}$ and Manfred Tscheligi ${ }^{1,2}$ \\ ${ }^{1}$ CURE - Center for Usability Research \& Engineering, Vienna, Austria \\ (busch, schrammel, tscheligi) @cure.at \\ ${ }^{2}$ University of Salzburg, ICT\&S Center, Salzburg, Austria \\ manfred.tschligiesbg.ac.at
}

\begin{abstract}
In this study we develop and validate an inventory for measuring persuadability to selected persuasive strategies. The development of the initial inventory was successful by means of internal consistency and item-scale correlation for the persuasive strategies rewards, competition, social comparison, trustworthiness and social learning. The inventory can be used to estimate susceptibility to persuasive strategies to personalize persuasive technology according to the users' personality based on self-reports. This can help system designers to make informed design decisions and to adapt persuasive technology.
\end{abstract}

\section{Introduction}

Persuasive Technology has been in the focus of HCI for over a decade - getting popular with Fogg's [1] Persuasive Technology: Using Computers to Change What We Think and Do in 2002. Different persuasive strategies such as e.g. rewards or social comparison have been suggested to be used in such technology. These persuasive strategies are supposed to change human attitudes and behavior. Innovative methods have been used to evaluate the effect of these persuasive technologies [2]. However, there are individual differences in the susceptibility to different persuasive strategies called persuadability [3][4][5][6].

To create personalized persuasive technology (which is expected to have greater impact than not-personalized technology), it is necessary to be able to estimate the susceptibility of a person to different persuasive strategies (persuadability) - this is also referred to as "Persuasion Profiling" [4]. As a contribution to improve the design of persuasive technology, we develop and initially validate scales to measure persuadability for selected persuasive strategies for which specific psychometric inventories do not exist yet, namely: Rewards, competition, social comparison, trustworthiness, simulation, reduction and social learning, based on a collection by Torning and Oinas-Kukkonen [7]. The initial scale development was successful (by means of internal consistency and item-scale correlation) for all scales except for: simulation and reduction.

adfa, p. 1, 2011.

(C) Springer-Verlag Berlin Heidelberg 2011 


\section{Related Work: Persuasive Technology, Persuadability and Scale Development}

A broad range of persuasive strategies have been identified, the most prominent are from Fogg (7 strategies) [1], Cialdini (6 strategies) [8] and a collection by Torning and Oinas-Kukkonen (28 strategies) [7]. Persuasive Technology is technology which incorporates one or more persuasive strategies. People differ in their susceptibility to different persuasive strategies. This leads to the assumption that personalized persuasive technology is more successful than persuasive technology that is intended to "fit for all" [4].

Persuadability in an HCI-context was introduced by Kaptein et al. [3] in 2009 and examined in several studies (see for example [4][5][9]). It is defined as the individuals' susceptibility to persuasive strategies and principles. Earlier, in the 1970's, Bauer defined persuadability in a marketing context as the disposition to shift one's attitude or judgment after being exposed to a persuasive communication [10]. Focused on persuasive systems, Kaptein, Lacroix and Saini [9] defined in 2010 persuadability as a tendency to comply to implementations of persuasive strategies. Several quantitative measures for the susceptibility to persuasive strategies have been developed, either in the domains of communication, cognition and social psychology (here called persuasibility instead of persuadability) [11][12] or in the domain of persuasion and HCI. Especially the work of Kaptein and colleagues (e.g. [3][4][5][6][9]) is of relevance. They developed a 7-Item persuadability questionnaire measuring persuadability to the persuasive strategies scarcity and authority [3], and further a persuadability questionnaire with six sub-scales reciprocity, scarcity, authority, commitment, consensus, liking, available as 12 and 32-item version [4]. The questionnaire items are statements that contain indicators for the susceptibility to single persuasive strategies, e.g. "When I am in a new situation, I look at others to see what I should do" focusing on the persuasive strategy consensus [4].

\section{Development and Validation of Persuadability Scales}

The goal of this work is to develop a reliable self-report inventory for important persuasive strategies for which no such questionnaires exist yet. In detail, the strategies rewards, competition, social comparison, trustworthiness, simulation, reduction and social learning are considered (chosen by experts - see next section). For scale development, we follow selected steps from a process that is proposed for construct measurement and validation procedures in management information systems: Definition of the construct, the generation of items to assess the construct (in section: 3.1 First Activity: Creation of the Scales), an assessment of content validity of the items, the collection of data to conduct a pretest and the scale purification and refinement (in section: 3.2 Second Activity: Initial Validation of the Scales) [13]. 


\subsection{First Activity: Creation of the Scales}

This section covers the first part of the scale development process [13], the item generation, and an initial assessment of the content validity of the items. We follow the best practice example of the development of the AttrakDiff-questionnaire [14] and conduct an HCI-expert workshop for the creation and expert validation of initial scales with 6 experts and a moderator. First, participants were introduced into concepts and definitions of persuasion and persuasive strategies in ICT and also into the concept of persuadability as (more or less) stable traits of personality that can predict the individuals' susceptibility to persuasive strategies. Then, the experts were presented the definitions of 28 persuasive strategies [7] (out of Torning \& Oinas-Kukkonen's collection) together with persuasive cues that can be implemented in interactive systems. As persuasive strategies are overall very abstract concepts and as some persuasive strategies are more abstract than others, participants had the task to choose a number of appropriate persuasive strategies as a basis for the creation of persuadability scales that fulfill the following criteria: Persuasive strategies can reasonably be translated into persuasive cues, these persuasive cues have nearly the same "estimated effect" on people and the susceptibility to these persuasive cues (persuadability) can be measured in a meaningful way by quantitative, verbal self-assessments. Per voting, experts chose 8 from 28 persuasive strategies that fulfilled the criteria best to be transferred into a questionnaire.

In a next step, the experts had to formulate self-assessment questionnaire items (in german) to assess the persuadability for the 8 chosen persuasive strategies. Experts were asked to develop precise items and to cover only one aspect per item. Overall, experts formulated 15 items for the scale simulation, 13 items for trustworthiness, 12 items for rewards, 12 items for social comparison, 11 items for social learning, 9 items for competition, 7 items for reduction and 3 items for recognition.

Then, an expert validation of the items was undertaken with the same experts that created the scales: Each expert had to rate each item of the 8 scales by means of fulfillment of the following criteria: If the item fits to the focal definition of the persuasive strategy dimension (content validity), if it covers only one specific aspect and if it can be assumed that the item represents an unidimensional scale together with all other items of that scale. Experts had to rate on a dichotomous scale: Fulfillment of the criteria $(+1)$ or no fulfillment of the citeria $(-1)$. In case of uncertainty, items did not have to be rated (0). For each scale, the 6 best rated items were chosen for a first version of the persuadability-inventory. As for the scale recognition only 3 items had been formulated, this scale was excluded from the inventory. The outcome of these steps is a first version of the persuadability-inventory with 7 scales with overall 42 items (see Table 1; all items).

\subsection{Second Activity: Initial Validation of the Scales}

This section describes the second part of the scale development process [13], a first item analysis (reliability by means of internal consistency and item-scale correlations) 
and the refinement of the scales. The items were administered in an online-study. First, participants were shortly introduced into the topic of the study and then administered the first version of the persuadability-inventory (see Table 1; all items) with a randomized order (to avoid an item-order bias). Answer format was a nine-point rating scale, ranging from $9=$ Fully agree to $1=$ Fully disagree . Participants were asked for sex, age and education.

We analyzed data from $n=167$ participants $(49,1 \%$ male; mean age: $M=37,6$; $S D=$ 14,4; education levels: 6,6\% secondary school, $15,6 \%$ apprenticeship, 41,3\% ALevels, $8,4 \%$ bachelor's degree, $26,3 \%$ master's degree, $1,8 \% \mathrm{PhD}$ level). For estimating reliability (internal consistency) we calculated Cronbachs Alpha. Also, we calculated corrected item-scale-correlations (ISC). All internal consistencies can be seen as sufficiently high to ensure reliability (over or near 0.7), except for simulation and reduction. Additionally, all ISCs of these two scales are under the commonly accepted critical value of 0.3. As a consequence, these two scales were skipped from the questionnaire. From the remaining scales, we eliminated all items with ISCs under 0.3. Although the Cronbachs Alpha of the trustworthiness-scale is below 0.7 (.472), its internal consistency is likely to increase after elimination of the items with ISCs under 0.3 (this new internal consistency has to be estimated with a different sample it is not appropriate to estimate it again with the same sample). The outcome of these steps is an iterated second version of the persuadability-inventory with 5 scales and overall 25 items (see Table 1; removed items are greyed out, items indicated with (r) have to be reversed before calculating an overall score). Scores resulting from questionnaire can be interpreted like this: Participants having higher scores in one or more of the scales are expected to be more susceptible to these specific persuasive strategies.

\section{Discussion and Future Work of the Scale Development}

This paper has shown the initial development (creation, expert validation, item analyis by means of internal consistency in online study) of 5 scales for measuring persuadability. 2 scales did not show sufficient internal consistency and some items showed low item-scale correlations. The 5 successfully developed scales can be used to estimate users susceptibility to certain persuasive strategies. Designers of persuasive technology can identify their intended user groups and use the questionnaire to extract the most effective persuasive strategies to be incorporated in the technology. However, a limitation of this work is that self-report questionnaires are highly susceptible to socially desirable answers and have disadvantages against other methods: Kaptein and Eckles have shown in 2012 [6] that these meta-judgemental measures of personality do a poor job in explaining heterogeneity of responses to persuasive strategies, but can - in addition to demographics and operative measures - still provide additional information. This opens a future challenges in improving these meta-judgemental measures until they can fruitfully complement or even compete other source of data. 
Table 1. The persuadability inventory (Final scales and items in black, removed ones in grey)

\begin{tabular}{|c|c|}
\hline Rewards (.818) (Cronbachs Alpha) & ISC \\
\hline 1. It is important to me that my actions are rewarded. & .593 \\
\hline 2. It is important for me to see my success before me. & .394 \\
\hline 3. I put more ambition into something, if I know I am going to be rewarded for it & .669 \\
\hline 4. I do more work, when I know that I will get something for it (something materialistic). & .673 \\
\hline 5. I am willing to change myself if I get rewarded. & .478 \\
\hline 6. Rewards motivate me. & .722 \\
\hline \multicolumn{2}{|l|}{ Competition (.658) } \\
\hline 7. I push myself hard, when I am in competition with others. & .465 \\
\hline 8. I would like to participate in Quiz shows, where I need to assert myself against other people. & .381 \\
\hline 9. Generally I am more ambitious than other people around me. & .407 \\
\hline 10. I am afraid to be seen as a loser. & .109 \\
\hline 11. It is important to me to be better than other people. & .592 \\
\hline 12. I like competitive sports (for example racing). & .476 \\
\hline \multicolumn{2}{|l|}{ Social comparison $(.758)$} \\
\hline 13. It is important to me to be equal in comparison to others & .497 \\
\hline 14. I like to compare myself to other people. & .624 \\
\hline 15. Before I do something, I want to know how other people have done it, so I can feel & .438 \\
\hline 16. It is important to me to know what other people are doing. & .522 \\
\hline 17. It is important to me, what other people think of me. & .508 \\
\hline 18. I adapt my style to the way my friends dress. & .400 \\
\hline \multicolumn{2}{|l|}{ Trustworthiness (.472) } \\
\hline 19. I think carefully about if I trust a system before I use it. & .319 \\
\hline 20. I trust information better where the source is specified. & .306 \\
\hline 21. I trust the information that I receive from the media. ( $\mathrm{r}$ ) & .058 \\
\hline 22. I listen more to a person when I know I can trust her/him. & .185 \\
\hline 23. It is important for me to be precisely informed about things that I need to do, before I do them. & .446 \\
\hline 24. I follow the advice from people that I believe are trustworthy. & .139 \\
\hline \multicolumn{2}{|l|}{ Simulation (.368) } \\
\hline 25. I often imagine how the earth will look like in the future. & .266 \\
\hline 26. I often imagine how it would be to look differently. & .088 \\
\hline 27. I like it when things are well illustrated, so I can get a better picture of things. & .170 \\
\hline 28. I find it interesting to know how things work. & .119 \\
\hline 29. It is important to me to see what influence my actions have on my surroundings. & .151 \\
\hline 30. I change my behavior more, when the results of that change are well illustrated. & .275 \\
\hline \multicolumn{2}{|l|}{ Reduction (.361) } \\
\hline 31. I take a detour when I go shopping, if it helps me save money. & .043 \\
\hline 32. When the operation of a device is problematic and complicated, I do not use it. (r) & .115 \\
\hline 33. I need clear facts to make a decision. & .170 \\
\hline 34. When I see the benefit of an action, I am more willing to perform this action. & .263 \\
\hline 35. I'm easily willing to follow an instruction that is clear and simple. & .264 \\
\hline 36. I prefer to make my decisions using precise information. & .273 \\
\hline \multicolumn{2}{|l|}{ Social Learning (.707) } \\
\hline 37. I often modify myself to other people. & .617 \\
\hline 38. I ask for advice from other people, before I make a decision. & .319 \\
\hline 39. I adopt my behavior quick to the model of other people. & .581 \\
\hline $\begin{array}{l}\text { 40. When I don't know something, I rather look on the internet or in books, than rely on advice from } \\
\text { other people. }\end{array}$ & .029 \\
\hline 41. I adapt my behavior to other people around me. & .594 \\
\hline 42. I take other people as role models for new behaviors. & .534 \\
\hline
\end{tabular}


Acknowledgments. This work was partially funded by the European Union Seventh Framework Programme (FP7) under grant 288466 (PEACOX; http://www.projectpeacox.eu) and by the Austrian project "AIR - Advanced Interface Research" funded by the Austrian Research Promotion Agency (FFG), the ZIT Center for Innovation and Technology and the province of Salzburg under contract number 825345 .

\section{References}

1. Fogg, B.J.: Persuasive Technology - Using Computers to Change What We Think and Do. Morgan Kaufmann (2002).

2. Döbelt, S., Schrammel, J., Subasi, Ö., Tscheligi, M.: Applying the Implicit Association Test for the Evaluation of Persuasive Technology. Persuasive Technology - Poster Proceedings (2010).

3. Kaptein, M., Markopoulos, P.: Can you be persuaded? individual differences in susceptibility to persuasion. INTERACT (2009).

4. Kaptein, M.C.: Personalized persuasion in Ambient Intelligence. Journal of Ambient Intelligence and Smart Environments. (2012).

5. Kaptein, M., De Ruyter, B., Markopoulos, P., Aarts, E.: Adaptive Persuasive Systems: A Study of Tailored Persuasive Text Messages to Reduce Snacking. ACM Transactions on Interactive Intelligent Systems (2012).

6. Kaptein, M., Eckles, D.: Heterogeneity in the Effects of Online Persuasion. Journal of Interactive Marketing. 26, (2012).

7. Torning, K., Oinas-Kukkonen, H.: Persuasive System Design: State of the Art and Future Directions. Persuasive Technology (2009).

8. Cialdini, Robert, B.: Harnessing the Science of Persuasion. Harvard Business School Publishing (2001).

9. Kaptein, M.C., Lacroix, J., Saini, P.: Individual differences in persuadability in the health promotion domain. Persuasive Technology (2010).

10.Bauer, R.: Self-Confidence and Persuadability: One More Time. Journal of Marketing Research. 7, (1970).

11.Nahemow, L., Bennett, R.: Conformity, persuasibility and counternormative persuasion. Sociometry. 30, (1967).

12.Cacioppo, J., Petty, R., Kao, C.: The efficient assessment of need for cognition. Journal of Personality Assessment. 48, (1984).

13.MacKenzie, S.B., Podsakoff, P.M., Podsakoff, N.P.: Construct measurement and validation procedures in MIS and behavioral research: integrating new and existing techniques. MIS Quarterly. 35, (2011).

14.Hassenzahl, M., Burmester, M., Koller, F.: AttrakDiff: Ein Fragebogen zur Messung wahrgenommener hedonischer und pragmatischer Qualität. Mensch \& Computer. 187-196 (2003). 